\title{
The Influence of Couple Stress Components and Electrostatic Actuation on Free Vibration Characteristics of Thin Micro-Plates
}

\author{
Amir R. Askari, Masoud Tahani \\ Department of Mechanical Engineering, Faculty of Engineering, Ferdowsi University of Mashhad, Mashhad, Iran
}

\begin{abstract}
The present paper deals specifically with the frequently asked question of are there any differences between classical non-actuated mode-shapes of plate-type MEMS and the electrically deformed ones obtained by the non-classical modified couple stress theory. To this end, a Kirchhoff's thin plate model together with the modified couple stress theory is considered to extract the eigenvalue problem which governs on free vibrations of the system. Using the free vibration equation of the system, it is proved that neither the electrostatic actuation nor the couple stress components influence on linear mode-shapes of the system. To emphasis on the validity of the present proof, the extended Kantorovich method is employed to provide analytical solutions for an electrically actuated micro-plate with clamped boundary conditions. The solutions, whose accuracy is verified by available results in the literature, also indicate that the linear mode-shapes of an electrically actuated micro-plate are totally independent from the influences of size and electrical loading.
\end{abstract}

\section{Introduction}

Technology of micro-electro-mechanical systems (MEMS) has experienced many improvements in recent years. The building blocks of these systems are some sort of electrically actuated mechanical structures such as bars, beams or plates [1]. In general, an electrically actuated micro-plate is a conductive and elastic thin plate suspended over a stationary rigid electrode and deflects toward its substrate by applying the external voltage [2]. One of the most important phenomena associated with electrically actuated micro-plates is pull-in instability which occurs when the input voltage reaches a critical value called pull-in voltage. In this manner, the elastic restoring force of the micro-plate cannot resist against the Coulomb attraction and it suddenly collapses toward the substrate underneath it [3].

Due to the coupling between electrical and structural physics as well as the strong non-linearities arising in the field of electrically actuated micro-structures, modelling of these systems is so challenging [4]. To date, variety of mathematical procedures has been employed to uncover the static and dynamic behaviors of such systems [5-7]. Amongst all of these mathematical techniques, reduced order modelling does not suffer from long run-time and has a unique advantage in providing the ability of presenting analytical solutions for MEMS problems [3]. In general, creating reduced order models (ROMs) eliminates the spatial dependence in the governing equations and performs a transformation from the physical coordinates of the device to a set of generalized coordinates. In this way, the governing differential equations of equilibrium are reduced to a set of algebraic equations which can be solved more easily than the governing equations themselves. It is to be mentioned here that utilizing the Galerkin weighted residual method for eliminating the spatial dependence is so usual in the creation of ROMs [3]. In this way, it is proved that employing linear mode-shapes of the system accelerates the convergence of the procedure and significantly reduces the computational costs $[2,8,9]$.

In recent years, it has experimentally been observed that the mechanical behavior of structures in small scales significantly depends on their size [10-13]. In spite of the fact that the classical continuum mechanics is the most common theory for investigating the mechanical behavior of structures, it is incapable of capturing their sizedependent behavior at micron and sub-micron scales. To remove this incapability of classical theory (CT), some higher-order theories of elasticity, which account for the effects of higher-order stress and strain components in the strain energy expression, are employed. Amongst all of the size-dependent higher-order theories, the modified couple stress theory (MCST), due to its simplicity and accuracy, has been frequently utilized to study the sizedependent behavior of MEMS devices [14-18].

As it was mentioned above, employing the linear mode-shapes of the system in the creation of ROMs significantly accelerates the convergence of the procedure. Hence, the main object of the present paper is to investigate the effects of size and electrostatic loading on the free vibration characteristics of electrically actuated thin micro-plates as one of the most important building blocks of MEMS devices. To this end, the size-dependent 
Kirchhoff thin plate model [19] is employed to develop the equation which governs on the linear free vibration of the system. Using this governing equation, it is proved that the micro-plate linear mode-shapes are totally independent of size and electrostatic loading. Also, it is shown that the MCST to CT frequency and pull-in voltage ratios of the system are totally independent of plate aspect ratio as well as its boundary condition and only depends on the Poisson ratio of the micro-plate. Finally, to emphasis on the independency of micro-plate mode-shapes from the influences of size and electrostatic loading, the extended Kantorovich method (EKM) is also employed to extract the size-dependent free-vibration characteristics of fully clamped micro-plates as the most applicable type of plate-based MEMS.

\section{Theoretical formulations}

Figure 1 shows a fully clamped electrically actuated rectangular micro-plate whose length, width, thickness and density are denoted by $b, a, h$ and $\rho$ respectively. A space-fixed Cartesian coordinate system is also attached to the center of the mid-plane of the micro-plate. That is, $\hat{x}, \hat{y}$ and $\hat{z}$ are the coordinates along the width, length and thickness, respectively. According to the MCSTbased size-dependent thin plate model presented by Tsiatas [19], the governing equation of motion takes the form [16]

$$
\rho h \frac{\partial^{2} \hat{w}}{\partial \hat{t}^{2}}+\left(\frac{E h^{3}}{12\left(1-v^{2}\right)}+\frac{E h l^{2}}{2(1+v)}\right) \nabla^{4} \hat{w}-F_{\mathrm{es}}=0
$$

where E, $v, l$ and $\hat{w}$ are the Young modulus, Poisson ratio, material length scale parameter and transverse deflection of the micro-plate, $\hat{t}$ is time and $\nabla^{4}$ operator refers to

$$
\nabla^{4}=\left(\frac{\partial^{4}}{\partial \hat{x}^{4}}+2 \frac{\partial^{4}}{\partial \hat{x}^{2} \partial \hat{y}^{2}}+\frac{\partial^{4}}{\partial \hat{y}^{4}}\right)
$$

The electrostatic actuation by polarized DC voltage $V_{\text {DC }}$ (i.e. $F_{\text {es }}$ ) can also be expressed as [8]

$$
F_{\mathrm{es}}=\frac{\varepsilon V_{D C}^{2}}{2(d-\hat{w})^{2}}
$$

in which $\varepsilon$ is the dielectric constant of medium. In addition $d$ is the initial gap between the non-actuated micro-plate and its substrate underneath it. Introducing the dimensionless variables $x=\hat{x} / a, y=\hat{y} / b$, $w=\hat{w} / d$ and $t=\hat{t} / T$ the non-dimensionalized form of the governing equation of motion takes the form

$$
\begin{aligned}
& \ddot{w}+\left(1+6 \alpha_{2}\right)\left(\frac{\partial^{4} w}{\partial x^{4}}+2 \alpha_{1}^{2} \frac{\partial^{4} w}{\partial x^{2} \partial y^{2}}+\alpha_{1}^{4} \frac{\partial^{4} w}{\partial y^{4}}\right) \\
& -\frac{\alpha_{3}}{(1-w)^{2}}=0
\end{aligned}
$$

where the dot super-script refers to partial derivative with respect to dimensionless time. Also, the time scale $T$, which is determined such that the coefficient of $\ddot{w}$ becomes unity, is defined as follows

$$
T=\sqrt{\frac{12 \rho a^{4}\left(1-v^{2}\right)}{E h^{2}}}
$$

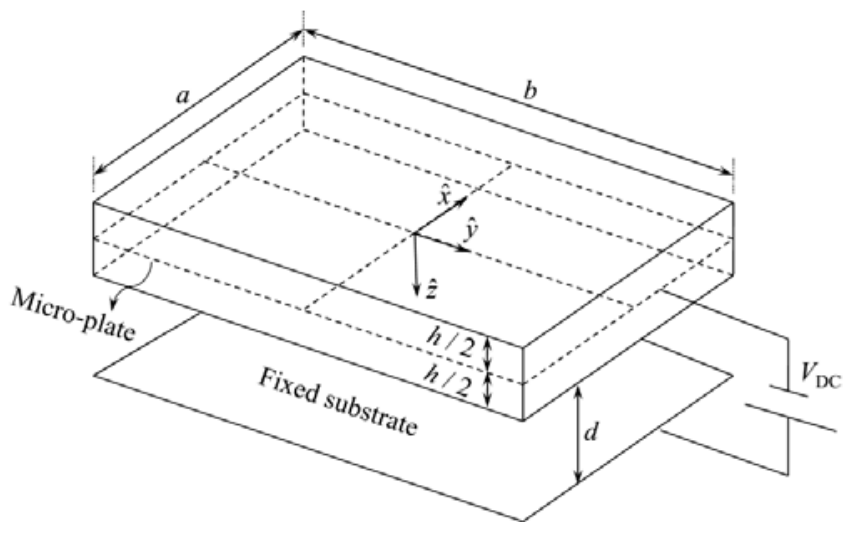

Figure 1. Schematic of an electrically actuated rectangular micro-plate.

The normalized parameters of the problem are also given by

$\alpha_{1}=\frac{a}{b}, \alpha_{2}=\frac{1-v}{(h / l)^{2}}, \alpha_{3}=\frac{6 \varepsilon a^{4}\left(1-v^{2}\right) V_{\mathrm{DC}}^{2}}{E h^{3} d^{3}}$

Assuming simple harmonic motion for the linearized system, the eigenvalue problem associated with Eq. (4) which governs on the free vibrations of a micro-plate can be obtained as

$\left(\frac{\partial^{4} \psi_{m n}}{\partial x^{4}}+2 \alpha_{1}^{2} \frac{\partial^{4} \psi_{m n}}{\partial x^{2} \partial y^{2}}+\alpha_{1}^{4} \frac{\partial^{4} \psi_{m n}}{\partial y^{4}}\right)-\lambda_{m n} \psi_{m n}=0$

where $\lambda_{m n}$ and $\psi_{m n}$ represent the eigenvalue of the Eq. (7) and its associated eigenfunction, respectively. It is to be noted that the eigenvalue of Eq. (7) relates to the natural frequencies of the micro-plate using Eq. (8) as

$$
\lambda_{m n}=\frac{\alpha_{3}+\omega_{m n}^{2}}{1+6 \alpha_{2}}
$$

As it is seen from Eq. (7), the size and electrostatic parameters (i.e. $\alpha_{2}$ and $\alpha_{3}$ ) are not appeared in this equation. Hence, it is obvious that variation of $\alpha_{2}$ and $\alpha_{3}$ does not change the eigenvalue and eigenfunction of this equation (i.e. $\lambda_{m n}$ and $\psi_{m n}$ ). Furthermore, according 
to Eq. (7), it can simply be concluded that $\lambda_{m n}$ and its associated eigenfunction (i.e. $\psi_{m n}$ ) will only be changed if the value of micro-plate aspect ratio (i.e. $\alpha_{1}$ ) is varied. It is worth noting to mention here that the independency of thin micro-plates linear mode-shapes from electrostatic excitation and couple stress components has also been observed numerically by Tahani et al. [16] using the finite element method.

As it was mentioned above, $\lambda_{m n}$ takes like values for micro-plates with same aspect ratio parameter. Hence, it is apparent that increasing the effect of size (i.e. the values of $\alpha_{2}$ ) increases all natural frequencies of the system. Also, increasing the electrostatic attraction by making an increase in the values of input voltages decreases all of its natural frequencies.

In view of Eq. (8), the ratio of size-dependent natural frequencies obtained by the MCST to those determined through the CT becomes

$$
\frac{\omega_{m n}^{\mathrm{MCST}}}{\omega_{m n}^{\mathrm{CT}}}=\sqrt{\frac{\left(1+6 \alpha_{2}\right) \lambda_{m n}-\alpha_{3}}{\lambda_{m n}-\alpha_{3}}}
$$

From Eq. (9), it is obvious that the MCST to CT natural frequency ratio in the absence of electrostatic field takes the form

$$
\frac{\omega_{m n}^{\mathrm{MCST}}}{\omega_{m n}^{\mathrm{CT}}}=\sqrt{1+6 \alpha_{2}}
$$

which is independent of the micro-plate aspect ratio as well as its boundary condition and only depends on the Poisson ratio of the micro-plate. It is worth noting that this important conclusion has also been reported numerically by Tsiatas [19] and analytically by Askari and Tahani [20] through the assumed mode method.

Let us consider Eq. (8) again. From this equation, one can write

$$
\omega_{m n}=\sqrt{\left(1+6 \alpha_{2}\right) \lambda_{m n}-\alpha_{3}}
$$

From Eq. (11), it is apparent that there exists a critical value for the electrostatic parameter in which the first natural frequency of the micro-plate is vanished. It is to be noted that this critical value of $\alpha_{3}$ can represent a good approximation for the pull-in parameter of the system $[8,9,16]$. Therefore, in view of Eq. (11), one can obtain the MCST to CT pull-in parameter ratio as

$$
\frac{\alpha_{3}^{\mathrm{MCST}}}{\alpha_{3}^{\mathrm{CT}}}=1+6 \alpha_{2}
$$

which is also independent of the micro-plate aspect ratio as well as its boundary condition and only depends on the Poisson ratio of the micro-plate. It is to be mentioned here that, according to Eq. (6), the MCST to CT pull-in voltage ratio also takes the form

$$
\frac{V_{\mathrm{PI}}^{\mathrm{MCST}}}{V_{\mathrm{PI}}^{\mathrm{CT}}}=\sqrt{1+6 \alpha_{2}}
$$

In the next section, employing the EKM, an analytical solution for micro-plates with fully clamped boundary conditions as the most applicable case in MEMS structures is provided to emphasis on the independency of micro-plate linear mode-shapes from the influences of size and electrical loading.

\section{Application of the EKM}

According to the general procedure of the EKM [21], the micro-plate mode-shape can be considered as a multiplication of two separable functions as

$$
\psi_{m n}(x, y)=f(x) g(y)
$$

where $f(x)$ and $g(y)$ are un-known functions in terms of $x$ and $y$ to be determined. Substituting the separated form of the micro-plate mode-shape into Eq. (7) and constructing the variational form of this equation, we obtain

$$
\begin{aligned}
& \int_{-1 / 2}^{1 / 2} \int_{-1 / 2}^{1 / 2}\left\{\left[\left(g \frac{d^{4} f}{d x^{4}}+2 \alpha_{1}^{2} \frac{d^{2} f}{d x^{2}} \frac{d^{2} g}{d y^{2}}+\alpha_{1}^{4} f \frac{d^{4} g}{d y^{4}}\right)\right.\right. \\
& \left.\left.-\lambda_{m n}(f g)\right] \delta(f g)\right\} d x d y=0
\end{aligned}
$$

The EKM is an iterative procedure in nature. Hence, it is assumed that $g$ is a prescribed known function satisfied the fully clamped boundary conditions. In this case, the variation of $\psi_{m n}$ would be just because of the variation of un-known function $f(x)$. Utilizing the fact that $\delta f$ takes arbitrary values except on the boundaries and employing the fundamental lemma of variational calculus [22], we obtain

$$
\frac{d^{4} f}{d x^{4}}-I_{1} \frac{d^{2} f}{d x^{2}}+\left(I_{2}-\lambda_{m n}\right) f=0
$$

where

$$
\begin{aligned}
& I_{1}=2 \alpha_{1}^{2} \frac{\int_{-1 / 2}^{1 / 2}\left(\frac{d g}{d y}\right)^{2} d y}{\int_{-1 / 2}^{1 / 2} g^{2} d y} \\
& I_{2}=\alpha_{1}^{4} \frac{\int_{-1 / 2}^{1 / 2}\left(\frac{d^{2} g}{d y^{2}}\right)^{2} d y}{\int_{-1 / 2}^{1 / 2} g^{2} d y}
\end{aligned}
$$

For the next iteration step, utilizing the prescribed known function $f$ obtained from Eq. (16), the ordinary differential equation (ODE) which governs on the unknown function $g$ can be determined as 


$$
\frac{d^{4} g}{d y^{4}}-I_{1}^{\prime} \frac{d^{2} g}{d y^{2}}+\left(I_{2}^{\prime}-\frac{\lambda_{m n}}{\alpha_{1}^{4}}\right) g=0
$$

where

$$
\begin{gathered}
I_{1}^{\prime}=\frac{2}{\alpha_{1}^{2}} \frac{\int_{-1 / 2}^{1 / 2}\left(\frac{d f}{d x}\right)^{2} d x}{\int_{-1 / 2}^{1 / 2} f^{2} d x} \\
I_{2}^{\prime}=\frac{1}{\alpha_{1}^{4}} \frac{\int_{-1 / 2}^{1 / 2}\left(\frac{d^{2} f}{d x^{2}}\right)^{2} d x}{\int_{-1 / 2}^{1 / 2} f^{2} d x}
\end{gathered}
$$

As it is seen, employing the EKM, the partial differential equation (PDE) which governs on the free vibration of the micro-plate is separated to two simple ODEs with constant coefficients. By choosing the initial guess function $g(y)=\left(4 y^{2}-1\right)^{2}$, the solution of Eq. (16) takes the form

$$
\begin{aligned}
f(x)= & C_{1} \sinh (\vartheta x)+C_{2} \cosh (\vartheta x) \\
& +C_{3} \sin (v x)+C_{4} \cos (v x)
\end{aligned}
$$

where

$$
\begin{gathered}
\vartheta=\sqrt{\frac{I_{1}}{2}+\sqrt{\lambda_{m n}+\left(\frac{I_{1}}{2}\right)^{2}-I_{2}}} \\
v=\sqrt{-\frac{I_{1}}{2}+\sqrt{\lambda_{m n}+\left(\frac{I_{1}}{2}\right)^{2}-I_{2}}}
\end{gathered}
$$

Imposing the boundary conditions and following some straight forward mathematical operations, we obtain

$$
\begin{gathered}
C_{2} \cosh \left(\frac{\vartheta}{2}\right)+C_{4} \cos \left(\frac{v}{2}\right)=0 \\
\vartheta C_{2} \sinh \left(\frac{\vartheta}{2}\right)-v C_{4} \sin \left(\frac{v}{2}\right)=0 \\
C_{1} \sinh \left(\frac{\vartheta}{2}\right)+C_{3} \sin \left(\frac{v}{2}\right)=0 \\
\vartheta C_{1} \cosh \left(\frac{\vartheta}{2}\right)+v C_{3} \cos \left(\frac{v}{2}\right)=0
\end{gathered}
$$

where Eqs. (22) and (23) are associated with symmetric and anti-symmetric mode-shapes, respectively. It is to be mentioned here that the eigenvalue of Eq. (16) can also be determined by equating the determinant of the coefficients of the sets of Eqs. (22) and (23), respectively, for symmetric and anti-symmetric mode-shapes. By continuing the iterative procedure, the solution of Eq. (16), which is presented in Eq. (20), can be utilized as the prescribed known function in Eq. (18) to find the unknown function $g(y)$ as

$$
\begin{aligned}
g(y)= & C_{1}^{\prime} \sinh \left(\vartheta^{\prime} y\right)+C_{2}^{\prime} \cosh \left(\vartheta^{\prime} y\right) \\
& +C_{3}^{\prime} \sin \left(v^{\prime} y\right)+C_{4}^{\prime} \cos \left(v^{\prime} y\right)
\end{aligned}
$$

where

$$
\begin{gathered}
\vartheta^{\prime}=\sqrt{\frac{I_{1}^{\prime}}{2}+\sqrt{\frac{\lambda_{m n}}{\alpha_{1}^{4}+\left(\frac{I_{1}^{\prime}}{2}\right)^{2}-I_{2}^{\prime}}}} \\
v^{\prime}=\sqrt{-\frac{I_{1}^{\prime}}{2}+\sqrt{\frac{\lambda_{m n}}{\alpha_{1}^{4}}+\left(\frac{I_{1}^{\prime}}{2}\right)^{2}-I_{2}^{\prime}}}
\end{gathered}
$$

It should be noted that, due to the similarity of Eqs. (16) and (18), their eigenvalue-eigenfunction equations will be the same (for more details see Ref. [20]). It has been shown that continuing the present procedure would leads to the convergence of the results which is so rapid and usually occurred after the second iteration step [20].

As it can be observed from Eqs. (16) - (25), the eigen value of Eq. (7) and the characteristics of the linear mode-shape of the micro-plate (i.e. $v, \vartheta, C_{i}, v^{\prime}, \vartheta^{\prime}$ and $\left.C_{i}^{\prime}(i=1,2,3,4)\right)$ only depend on the values of $I_{1}, I_{2}$, $I_{1}^{\prime}$ and $I_{2}^{\prime}$ which are independent of the size and electrostatic parameters. In other words, as it is expected, the eigenvalue and eigenfunction of Eq. (7) depend only on the micro-plate aspect ratio.

\section{Results and Discussion}

To validate the accuracy of the present EKM procedure, a convergence study is carried out in Table 1 for the first two eigenvalues of a square micro-plate. As it is seen from this Table, the convergence of the EKM is so rapid and usually occurs after the second iteration. It is noteworthy that, since the square root of the micro-plate eigenvalues are on the order of its natural frequencies, we have chosen to present them in Table 1 instead of the eigenvalues themselves to provide a better physical sense.

Table 1. Convergence of the first two eigenvalues of a square micro-plate.

\begin{tabular}{lcccc}
\hline $\begin{array}{l}\text { Number of } \\
\text { Iteration }(i)\end{array}$ & $\left.\sqrt{\lambda_{11}}\right|_{i, i-1}$ & $\left.\sqrt{\lambda_{11}}\right|_{i, i}$ & $\left.\sqrt{\lambda_{12}}\right|_{i, i-1}$ & $\left.\sqrt{\lambda_{12}}\right|_{i, i}$ \\
\hline 1 & 35.9995 & 35.9990 & 35.9995 & 73.5333 \\
2 & 35.9990 & 35.9990 & 73.4054 & 73.4054 \\
3 & 35.9990 & 35.9990 & 73.4054 & 73.4054 \\
4 & 35.9990 & 35.9990 & 73.4054 & 73.4054 \\
\hline
\end{tabular}

Verification is also performed here for the converged values of the first four eigenvalues of micro-plates with three different aspect ratios in Table 2 . The square roots 
of these eigenvalues are compared with those reported by Arenas [23] for large scale plates in the absence of the electrostatic field using the CT. It is to be noted that, according to Eq. (8), the square root of the micro-plate eigenvalues equals with its classical natural frequencies in the absence of electrostatic field. As it can be observed from Table 2, the present findings are in excellent agreement with those published by Arenas [23].

Table 2. Comparison of the first four eigenvalues for a microplate with three different aspect ratios.

\begin{tabular}{llcccc}
\hline$\alpha_{1}$ & & $\sqrt{\lambda_{11}}$ & $\sqrt{\lambda_{12}}$ & $\sqrt{\lambda_{21}}$ & $\sqrt{\lambda_{22}}$ \\
\hline 1 & Present & 35.9990 & 73.4054 & 73.4054 & 108.2359 \\
& Arenas [23] & 36.1087 & 73.7372 & 73.7372 & 108.8499 \\
0.5 & Present & 24.5812 & 31.8334 & 63.9853 & 71.0813 \\
& Arenas [23] & 24.6480 & 31.9618 & 64.1724 & 71.4252 \\
0.1 & Present & 22.4324 & 22.6112 & 61.7503 & 61.9810 \\
& Arenas [23] & 22.4419 & 22.6335 & 61.7650 & 62.0188 \\
\hline
\end{tabular}

As it is shown in two previous sections, the MCST to CT frequency and pull-in voltage ratios depend only on the Poisson ratio of the micro-plate. This fact is illustrated in Figure 2. Figure 2 depicts the MCST to CT frequency or pull-in voltage ratios versus the variation of the normalized length scale parameter (i.e. $h / l$ ) for three different Poisson's ratios. As it is seen from this figure, the effect of size on micro-plate natural frequencies and its pull-in stability threshold can usually be ignored for cases in which the ratio of micro-plate thickness to its material length scale parameter is larger than 10. In addition, increasing the values of Poisson's ratio of the micro-plate decreases the MCST to CT frequency and pull-in voltage ratios.

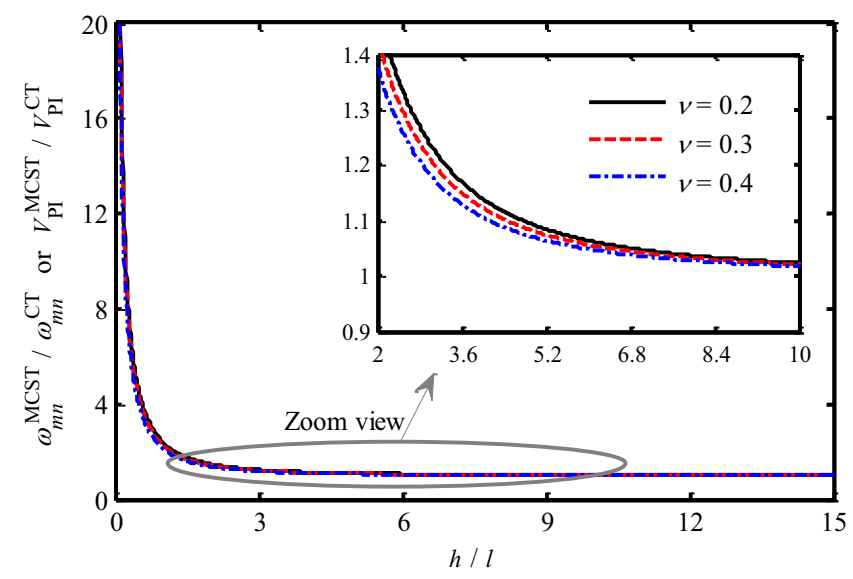

Figure 2. Influence of size on natural frequencies and pull-in instability threshold.

\section{Concluding remarks}

In the present paper, the influences of size and electrostatic actuation on free vibration characteristics of rectangular thin micro-plates were studied. It was proved that the linear mode-shapes of these structures are totally independent of size and electrostatic loading. Also, it was shown that the MCST to CT frequency and pull-in voltage ratios are totally independent of micro-plate aspect ratio as well as its boundary conditions and only depends on the Poisson ratio of the micro-plate. Furthermore, to emphasis on the independency of microplates linear mode-shapes from the effects of size and electrostatic loading, an analytical solution for microplates with fully clamped boundary conditions was extracted using the EKM. The accuracy of the EKM solution was also verified through the direct comparison between its findings and those published in the literature.

\section{References}

1. S. D. Senturia, Microsystem Design, Kluwer Academic Publishers, Dordrecht (2001)

2. R. C. Batra, M. Porfiri and D. Spinello, Smart Mater. Struct. 16, 23-31 (2007)

3. A. H. Nayfeh, M. I. Younis and E. M. AbdelRahman, Nonlinear Dynam. 41, (1), 211-236 (2005)

4. M. I. Younis, MEMS Linear and Nonlinear Statics and Dynamics, Springer, New York (2011)

5. C. Jin, Z. Jiang, and W. Xingtao, J. Micromech. Microeng. 14, 1, 57 (2004)

6. H. Sadeghian, G. Rezazadeh, and P. M. Osterberg, J. Microelectromech. S. 16, 6, 1334-1340 (2007)

7. J.-H. Kuang and C.-J. Chen, J. Micromech. Microeng. 14, 4, 647 (2004)

8. R. C. Batra, M. Porfiri, and D. Spinello, Int. J. Solids Struct. 45, 3558-3583 (2008)

9. R. C. Batra, M. Porfiri and D. Spinello, j. Sound Vib. 309, 600-612 (2008)

10. N. A. Fleck, G. M. Muller, M. F. Ashby, and J. W. Hutchinson, Acta Metall. Mater. 42, 475-487 (1994)

11. J. S. Stölken and A. G. Evans, Acta Mater. 46, 51095115 (1998)

12. A. C. M. Chong and D. C. C. Lam, J. Mater. Res. 14, 4103-4110 (1999)

13. A. W. McFarland and J. S. Colton, J. Micromech. Microeng. 15, 1060-1067 (2005)

14. M. Rahaeifard, M. H. Kahrobaiyan, M. Asghari, and M. T. Ahmadian, Sensor Actuat. A-Phys. 171, 370374 (2011)

15. A. Mohsenzadeh, M. Tahani, and A. R. Askari, J. Nanoscience 2015, 9 (2015)

16. M. Tahani, A. R. Askari, Y. Mohandes, and B. Hassani, Int. J. Mech. Sci. 94-95, 185-198 (2015)

17. A. R. Askari and M. Tahani, Appl. Math. Model. 39 (2), 934-946 (2015)

18. K. F. Wang, T. Kitamura, and B. Wang, Int. J. Mech. Sci. 99, 288-296 (2015)

19. G. C. Tsiatas, Int. J. Solids Struct. 46, 2757-2764 (2009)

20. A. R. Askari and M. Tahani, J. Mech. Sci. Technol. 29, 5, 2135-2145 (2015)

21. A. D. Kerr, Int. J. Solids Struct. 5, 559-572 (1969)

22. J. N. Reddy, Energy Principles and Variational Methods in Applied Mechanics, John Wiley \& Sons, New York (2002)

23. J. P. Arenas, J. Sound Vib. 266, 912-918 (2003) 